\title{
Melioidosis in a Patient with Type I Diabetes Mellitus - A Case Report
}

\author{
TASNIMAAHMED ${ }^{1}$, MD. ABID HOSSAIN MOLLAH ${ }^{2}$, FAUZIA MOHSIN $^{3}$, JEBUN NAHAR ${ }^{4}$, ABU TALHA $^{5}$
}

\begin{abstract}
Melioidosis is a relatively uncommon but fatal infectious disease caused by the gram negative bacterium, Burkholderia pseudomallei which is usually found in the wet soil, mud and pooled surface water in tropic and subtropics. Documented reports of melioidosis from Bangladesh have been few and sporadic. All of the reported cases in Bangladesh were on adult. Melioidosis is commonly associated with underlying diseases like diabetes mellitus, renal diseases and immunodeficiency disorders. Early and correct diagnosis is important, as mortality in untreated melioidosis is high. Our patient, an eleven years old boy, a known case of diabetes mellitus, presented with fever for one month associated with right sided neck swelling, abdominal distension \& pain for 10 days. He had an abscess on the right side of the neck and hepatosplenomegaly. Ultrasonography of abdomen showed multiple abscesses in the liver and spleen. Culture of pus drained from the neck abscess revealed growth of Burkholderia pseudomallei. After getting parenteral antibiotics and insulin, his abscesses gradually resolved and cured completely.
\end{abstract}

Keyword: Melioidosis, Type I Diabetes Mellitus, Children.

\section{Introduction}

Melioidosis is an infectious disease caused by the gram-negative Burkholderia pseudomallei bacillus. It is associated with significant mortality due to an early onset of fulminant sepsis. ${ }^{1}$ The disease is endemic in Southeast Asian countries and in Northern Australia. It is now an emerging infection in Bangladesh. ${ }^{2}$ The organism is found in wet soil, mud and pooled surface water in the tropics and subtropics. Transmission to human is by inoculation, inhalation or ingestion and causes disease mostly in the immunocompromised conditions like diabetes mellitus or any other chronic illnesses. ${ }^{1,2}$ Documented reports of melioidosis from Bangladesh have been few and sporadic. The first culture proven case was reported from Bangladesh in

1. Registrar, Dept. of Paediatrics, BIRDEM General Hospital, Dhaka

2. Professor \& Head, Dept. of Paediatrics, BIRDEM General Hospital, Dhaka

3. Professor, Dept. of Paediatrics, BIRDEM General Hospital, Dhaka

4. Associate Professor, Dept. of Paediatrics, BIRDEM General Hospital, Dhaka

5. Assistant Registrar, Dept. of Paediatrics, BIRDEM General Hospital, Dhaka

Correspondence to: Dr. Tasnima Ahmed, Registrar, Dept. of Paediatrics, BIRDEM General Hospital, 1/A Ibrahim Sharani, Shegun Bagicha, Dhaka-1000, Email:tasnima695@ gmail.com, phone: 01845017199

Received: 20 December 2017

Accepted: 13 March 2018
1988. ${ }^{3}$ Later on, in 2001 another case of melioidosis in an adult with diabetes mellitus was reported from BIRDEM hospital. ${ }^{4}$ Thereafter case reports of melioidosis have been published sporadically from Bangladesh. ${ }^{5}$

With the existing geographical and climatic conditions and a susceptible population, Bangladesh seems to have ideal conditions for endemicity of this disease. Its true prevalence in Bangladesh is not known, due to misdiagnosis and under reporting. South Asia, including Bangladesh, is predicted to bear $44 \%$ of the overall burden. ${ }^{5}$

The classical presentation of this condition are pneumonia and multiple abscesses, with a mortality rate of up to $40 \%$. Melioidosis has a myriad of clinical manifestations and can present either as acute fulminant infection or chronic indolent debilitating illness. ${ }^{6}$ However, it has also been reported in asymptomatic patient. ${ }^{6}$ It also found in neonates recognised with possible feto-maternal transmission. ${ }^{7}$ We report a diabetic child hailing from Sherpur who has melioidosis.

\section{Case Report}

An eleven years old boy, hailing from Sherpur presented with the complaints of low grade fever for one month, right sided neck swelling and abdominal distention with 
pain for ten days. He was known case of diabetes mellitus for two years and he was on subcutaneous short acting and intermediate acting insulin, but he did not come for follow up regularly and his glycaemic control was poor. His father is a farmer \& he used to work at field with his father. When he presented to us, he was grossly emaciated, undernourished, febrile, mildly pale. There was an abscess on the right side of the neck which was about $7.5 \mathrm{~cm} \times 6.8 \mathrm{~cm}$, tender, soft on palpation. His abdomen was hugely distended due to grossly enlarged liver, which was tender with irregular surface and variable consistency. There was also mild splenomegaly. There was no ascites. Regarding anthropometric measurement he was underweight (height-138cm, weight-22kg, BMl-11.5 Kg/m²).
Some investigations were done, that revealed moderate anaemia, neutrophilic leucocytosis with thrombocytosis. Blood glucose was high $(25.5 \mathrm{mmol} /$ I). Liver functions were normal except there was hypoalbuminemia(Serum albumin- $2.1 \mathrm{gm} / \mathrm{dl}$ ). Creactive protein was $115 \mathrm{mg} / \mathrm{l}$. Blood culture revealed growth of pseudomonas, which was sensitive to ceftazidime, meropenem \& imipenem. USG of abdomen showed enlarged liver with multiple abscess of different size involving both lobes \& multiple small abscess in spleen (Figure-I). Culture and sensitivity of pus drained from the neck abscess revealed growth of Burkholderia pseudomallei, sensitive to ceftazidime, ceftriaxone, ciprofloxacin (Figure-2).

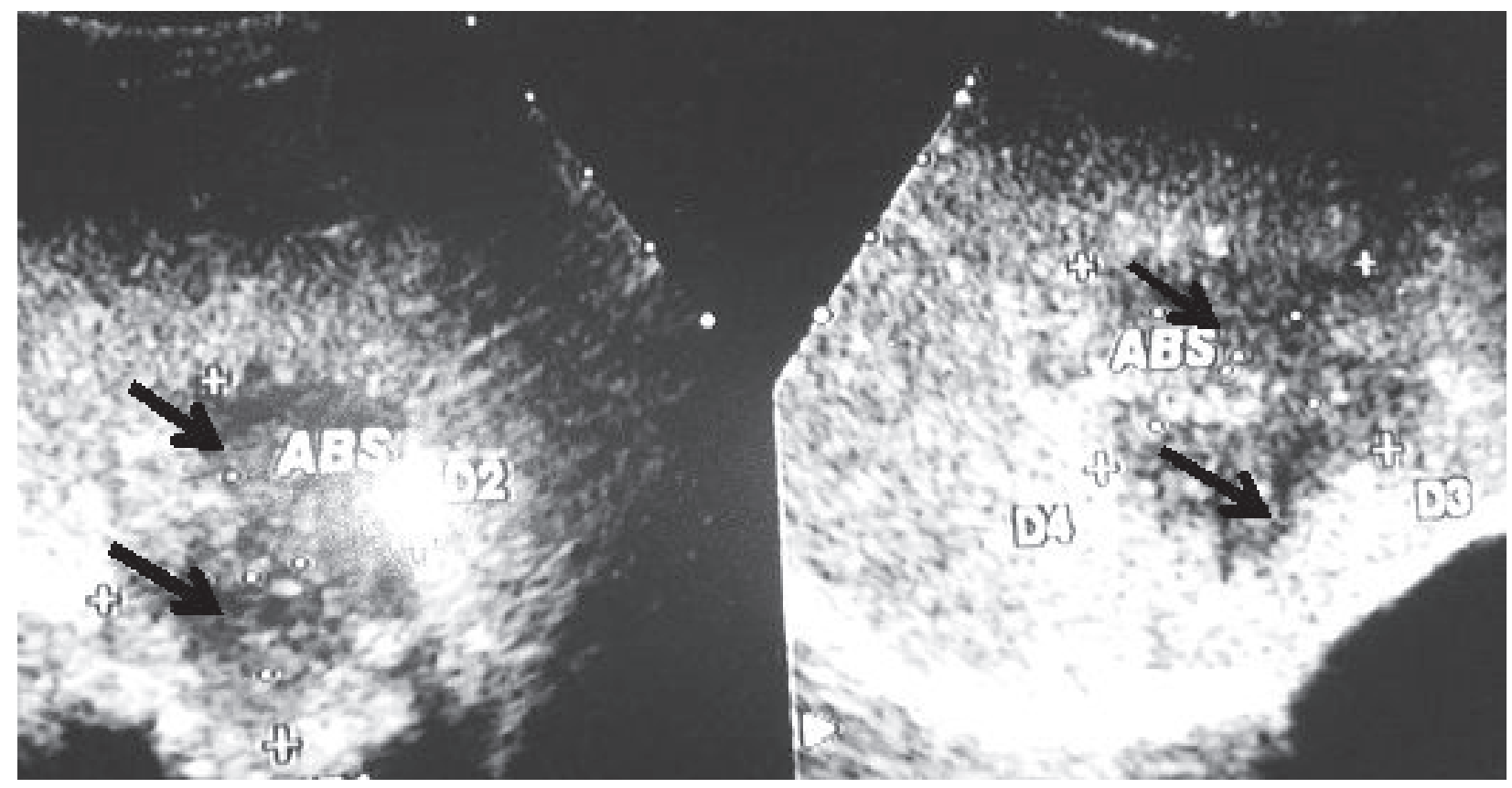

Fig.-1: USG of abdomen showed multiple abscess of different size involving both lobes of the liver

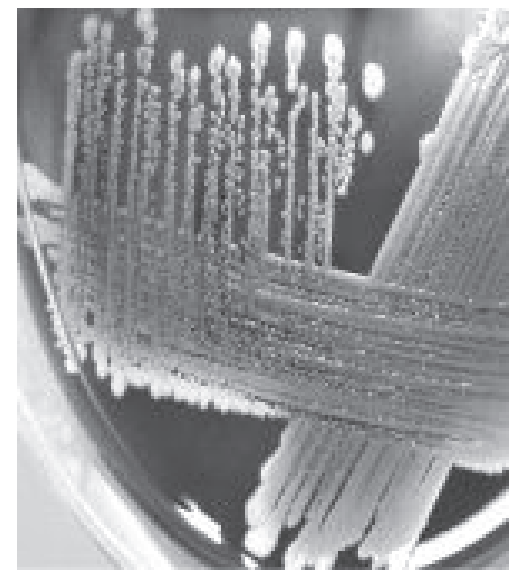

Fig.-2: Culture of pus drained from the neck abscess which revealed growth of Burkholderia pseudomallei
He was diagnosed as a case of Diabetes Mellitus with Sepsis with Melioidosis and managed with inj ceftazidime for three weeks. Blood glucose was strictly maintained with insulin. His general condition gradually improved and his abscesses gradually resolved after three weeks of injectable antibiotics. Then he was discharged with oral Cortrimoxazole for three months and advised for follow up.

\section{Discussion}

Melioidosis is an emerging infection in Bangladesh. It is still underreported due to its diverse clinical manifestations and inadequacy of conventional bacterial identification methods. ${ }^{8}$ Moreover, clinical, radiological and pathological similarities with 
tuberculosis may result in overlooking newly emerging diseases like melioidosis in tuberculosis endemic areas. ${ }^{9}$ Worldwide about $1,65,000$ people are diagnosed as case of melioidosis per year. ${ }^{8-10}$ Among them $15 \%$ account for paediatric melioidosis in endemic areas. ${ }^{10,11}$ Diabetes mellitus has been found to be one of the most frequent predisposing factor. Our patient was diabetic. Vidyalaxmi et al. found a correlation of $76 \%$ of DM and melioidosis. ${ }^{12}$ In Bangladesh, during the period from 1988 to 2016, twenty one cases of culture confirmed melioidosis have so far been reported. Almost all of them suffered from diabetes mellitus. The organism has a unique ability to bind human insulin, and this bacterial property underlie a remarkable biological and clinical relationship with important implications for diabetis. ${ }^{13}$

The presentation of melioidosis ranges from localized to systemic infection. Infection by the causative agent B. pseudomallei causes abscess formation in different organs of the body, such as lung, liver, prostate and soft tissues. It presents as a febrile illness, ranging from acute fulminant septicaemia to a chronic, debilitating localized infection. ${ }^{14}$

Mortality rate remains high, even with aggressive antibiotic therapy. In endemic areas mortality rate ranged from $19 \%$ to $68 \% .{ }^{4}$ It stresses the need for quick diagnosis and early initiation of appropriate treatment. The antibiotic of choice for melioidosis is ceftazidime. Imepenem is safe and effective and considered as alternatives to ceftazidime. Since relapse rates are high, initial parenteral treatment followed by maintenance therapy with Cortrimoxazole or Doxycycline or co-Amoxyclav for 12 to 20 weeks are recommended. ${ }^{14}$

\section{Conclusion}

Melioidosis can be presented as multiple liver abscess and abscess in different part of the body. It is more common in immunocompromised patient like diabetes mellitus. Early diagnosis and prompt treatment with appropriate antibiotic can save a patient from this deadly infection.

\section{References}

1. White NJ. Melioidosis. Lancet 2003;361:1715-22.

2. Limmathurotsakul D, Dance DAB, Wuthiekanun $\mathrm{V}$, Kaestli M, Mayo M, Warner J, et al. Systematic review and consensus guidelines for environmental sampling of Burkholderia pseudomallei. PLoS Negl Trop Dis 2013; 7(3): 2105.

3. Struelens MJ, Mondol G, Bennish M, Dance DAB. Melioidosis in Bangladesh: a case report. Trans R Soc Trop Med Hyg 1988; 82: 777-8.

4. Nazimuddin K, Hossain M, Mansur A, Haq JA, Khan AR. Melioidosis- a case report. J of Bangladesh College of Physicians \& Surgeons 2001; 19(2): 71-4.

5. Barai L, Jilani SA, Haq JA. Melioidosis-Case reports and review of cases recorded among Bangladeshi population from 1988-2014. Ibrahim Med Coll J 2014;8(1):25-31.

6. Dayasiri M B K C, Mudiyanse R M, Kudagmmana H D W S, Rafia M I, Jeyaratnasingham C. Melioidosis manifesting as severe emaciation and clinically indolent liver abscess in a child with Beta thalassaemia major. Sri Lanka Journal of Child Health 2016; 45:130-33.

7. Halder D, Zainal N, Haq JA. Neonatal meningitis and septicemia caused by Pseudomonas pseudomallei. Annals of Tropical Pediatrics 1998; 18: 161-64.

8. Afroze SR, Barai L, Rahim MA et al. Sociodemographic, Clinical and Laboratory Characteristics of Melioidosis: Four-Year Experience of Managing Consecutive 11 Cases in a Tertiary Care Hospital of Bangladesh. BIRDEM Med J 2017;7(1):28-37.

9. Adhikary P, Selim S, Uddin N et al. Melioidosis Mimicking tuberculosis in an endemic zone. SM Journal of Infec Dis 2016;1:1002.

10. Lumbiganon $\mathrm{P}$, chotechuangnirun $\mathrm{N}$, Kosalaraksa $P$, et al. Localized melioidosis in children in Thailand: treatment and long-term outcome. J Trop Pediatr 2011;57:185-91.

11. Foong YW, Tan NW, Chong CY, Thoon Kc, Tee NW, Koh MJ. Melioidosis in children. Int J Dermatol 2015;54:929-38.

12. Vidyalakshmi KS, Shrikala B, Bharathi B, Suchitra U. Melioidosis: An under-diagnosed entity in western coastal India: A clinicomicrobiological analysis. Indian J Med Microbiol 2007;25:245-8.

13. Cheng AC, Currie BJ. Melioidosis: epidemiology, pathophysiology and management. Clin Microbiol Rev 2005; 18(2): 383-416.

14. Zueter1 AR, Yean CY, Abumarzouq M, Rahman ZA, Deris ZZ, Harun A. The epidemiology and clinical spectrum of melioidosis in a teaching hospital in a North-Eastern state of Malaysia: a fifteen year Review. BMC Infect Dis 2016; 16:33344. 